\title{
Singularly Perturbed Renormalization Group Method and Its Significance in Dynamical Systems Theory
}

\author{
Wenlei $\mathrm{Li}^{1}$ and Shaoyun $\mathrm{Shi}^{1,2, *}$ \\ ${ }^{1}$ School of Mathematics, Jilin University, Changchun 130012, P.R. China. \\ 2 State Key Laboratory of Automotive Simulation and Control, Jilin \\ University, Changchun 130012, P.R. China.
}

Received 16 April 2021; Accepted 23 July 2021

\begin{abstract}
In this paper, we mainly investigate three topics on the renormalization group (RG) method to singularly perturbed problems: 1) We will present an explicit strategy of RG procedure to get the approximate solution up to any order. 2) We will refer that the RG procedure can, in fact, be used to get the normal form of differential dynamical systems. 3) We will also present the approximating center manifolds of the perturbed systems, and investigate the long time asymptotic behavior by means of RG formula.
\end{abstract}

AMS subject classifications: 34D15, 34E05, 37D10, 37G05

Key words: Renormalization group method, normal form, center manifolds.

\section{Introduction}

Renormalization group ( $R G)$ method in the singular perturbation theory was originally introduced by Chen et al. [1,2] in 1980s, inspired from the classical renormalization idea in quantum mechanics [10]. The main goal of this method is to give a unified strategy to compute the effective approximate solution of different kinds of singular perturbation problems. So far, the RG method has been

${ }^{*}$ Corresponding author. Email addresses: 1 wlei@jlu.edu.cn (W. Li), shisy@jlu.edu.cn (S.Shi) 
turned out to be very useful in a large number of singular perturbed problems, such as secular problems, center manifolds etc. $[2,5,7,9,11,12,15,16,19]$.

Maybe the first rigorous investigation of RG method can be traced back to Ziane's consideration of following perturbed systems [22] in 1999:

$$
\left\{\begin{array}{l}
\dot{\mathbf{x}}+\frac{1}{\varepsilon} A \mathbf{x}=\mathbf{f}(\mathbf{x}), \\
\mathbf{x}(0)=\mathbf{x}_{0},
\end{array}\right.
$$

where $\mathbf{x} \in \mathbb{C}^{n}, \varepsilon$ is a small parameter, $A$ is a complex diagonalizable matrix, and $\mathbf{f}(\mathbf{x})$ is a polynomial nonlinear term. Based on the typically renormalization procedure, Ziane obtained two approximate results under certain assumptions, the corresponding strategy is unified, concise and effective. Following Ziane's formulation, RG method has been successfully used to analyze a large kinds of problems including the singular perturbed semi-linear PDE problems $[9,13,14,17,21]$.

In 2003, Temam and Wirosoetisno [20] considered a class of systems with the form

$$
\left\{\begin{array}{l}
\dot{\mathbf{x}}+\frac{1}{\varepsilon} L \mathbf{x}+A \mathbf{x}+B(\mathbf{x})=\mathbf{f}(t), \\
\mathbf{x}(0)=\mathbf{x}_{0}
\end{array}\right.
$$

where $L$ is a real antisymmetric matrix, $A$ is a positive-definite matrix, $\mathbf{f}(t)$ is given with $|\mathbf{f}|_{\infty}=\operatorname{esssup}_{0 \leq t \leq \infty}|\mathbf{f}(t)|$ finite, and $B(\mathbf{x})=\sum_{i=1}^{r} B_{i}(\mathbf{x})$, with $B_{i}(\mathbf{x})$ the $i$-linear and completely antisymmetric in $\mathbf{x}$, i.e., the inner product $\langle B(\mathbf{x}), \mathbf{x}\rangle=0$ for all $\mathbf{x} \in \mathbb{R}^{d}$. The authors presented an implicit procedure to obtain the approximate solution up to any order, they also made several dynamical analysis about the conservation, or dissipation of energy in different cases, which implies the simplicity of RG method compared with other ones.

In 2008, Chiba [5] considered another class of singular perturbation problems as the following form:

$$
\dot{\mathbf{x}}=\varepsilon \mathbf{g}(\mathbf{x}, t, \varepsilon), \quad \mathbf{x} \in U,
$$

where $U$ is an open set in $\mathbb{C}^{n}$ and the closure $\bar{U}$ is compact, $\mathbf{g}(\mathbf{x}, t, \varepsilon)$ is a vector field parameterized by $\varepsilon \in \mathbb{R}_{+}$. Inspired by the KBM theory, he presented a higher order RG theory for above system with a key assumption that the nonlinear terms are almost-periodic in $t$, and the set of corresponding Fourier exponents having no accumulation on $\mathbb{R}$. Moreover, his work turns out that, in many cases, RG theory can also lead to the existence of approximate invariant manifolds, inheritance of symmetries from those for the original equation to those for the RG equation, and unify traditional singular perturbation methods, such as the averaging method, the multiple time scale method and the center manifold reduction, etc. 
One common feature of above two results is that the RG strategies they used are the same as the one adopted in averaging method [18], which is, in fact, different to the typical RG idea, and only work on the KBM conditions. While, as it was presented in [2], there are many other kinds of problems, rather than KBM cases, that can also be solved by RG method, such as boundary layer problem [23]. Therefore, it is necessary to develop some more systematic and unified schemes for general perturbed problems.

However, from the original RG scheme, i.e., by introducing a free parameter to remove the secular terms in the naive expansion, it is hard to get a consistent and clear methodology that can be applied to other kinds of problems. In 2012, Kirkinis [11] pointed out that the RG method for asymptotic analysis is still in the early stages of development, and there are still many unsettled issues. One of the interesting questions raised by Kirkinis is, "Is there a consistent and clear methodology that one can apply to even linear, singularly perturbed PDEs?" The main goal of the present paper is to carry out the study on above questions. We will focus on the following $n$-dimensional differential system:

$$
\dot{\mathbf{x}}=A \mathbf{x}+\varepsilon \mathbf{f}(\mathbf{x}), \quad \mathbf{x} \in U,
$$

where " " means the derivative with respect to time $t, A$ is a complex matrix, assumed for simplicity to be diagonalizable, $U$ containing $\mathbf{0}$ is an open subset of $\mathbb{C}^{n}$, and $\mathbf{f}: U \rightarrow \mathbb{C}^{n}$ is a vector-valued nonlinear polynomial function, i.e., it can be rewritten as

$$
\mathbf{f}(\mathbf{x})=\sum_{\alpha \in \mathbb{N}^{n}, 2 \leq|\alpha| \leq N} \mathbf{f}_{\alpha} \mathbf{x}^{\alpha}
$$

with $\mathbf{f}_{\alpha} \in \mathbb{C}^{n}$ the vector valued coefficient of $\mathbf{x}^{\alpha}=x_{1}^{\alpha_{1}} \cdots x_{n}^{\alpha_{n}}$, and $|\alpha|=\alpha_{1}+\cdots+\alpha_{n}$. We remark that, with a time scaling, system (1.4) is, in fact, the same as (1.1). In 2008, Lee DeVille et al. [6] computed the approximate solution of (1.4) by classical RG method up to second order, and referred that the RG method can be used to compute the corresponding normal form of the given system. Here, we will develop a newly combined technique to give a systematic and explicit strategy of the RG method up to any order for the system (1.4), and give a complete consideration of its dynamical meaning in both aspects of normal form and center manifolds.

The article is organized as follows. In Section 2, we will present a systematic and explicit procedure of the RG method to obtain the asymptotic solution of (1.4) up to any order. In Section 4, the rigorous approximate estimates in different cases will be presented. In Section 5, the obtaining of the normal form will be naturally presented in any order. And we will give further consideration to the center manifolds approximation at the last section. 


\section{Renormalization group illustration}

In this section, we present an explicitly systematic RG procedure to get approximate solutions up to any order. Compared with the classical RG idea, our strategy can be viewed as a combined method with the free parameter and the higher order expansions.

Without loss of generality, we assume $A=\operatorname{diag}\left\{\lambda_{1}, \cdots, \lambda_{n}\right\}$. Let

$$
\mathbf{g}(t, \mathbf{x})=\mathbf{G}(t, \mathbf{x}, \mathbf{z}(t))
$$

with $\mathbf{G}(t, \mathbf{x}, \mathbf{z}): \mathbb{R} \times U \times \mathbb{C}^{2 n} \rightarrow \mathbb{C}$ a vector-valued polynomial function, and $\mathbf{z}(t)=$ $\left(e^{\lambda_{1} t}, \cdots, e^{\lambda_{n} t}, e^{-\lambda_{1} t}, \cdots, e^{-\lambda_{n} t}\right)$. Then it is not difficult to see that $\mathbf{g}(t, \mathbf{x})$ can be rewritten as

$$
\mathbf{g}(t, \mathbf{x})=\overline{\mathbf{g}}(\mathbf{x})+\mathbf{g}_{N}(t, \mathbf{x})+\mathbf{g}_{N T}(t, \mathbf{x})
$$

with $\mathbf{g}(\mathbf{x})$ the terms independent of time $t$ in $\mathbf{g}(t, \mathbf{x}), \mathbf{g}_{N T}(t, \mathbf{x})$ the terms having polynomials in $t$, and $\mathbf{g}_{N}(t, \mathbf{x})=\mathbf{g}(t, \mathbf{x})-\overline{\mathbf{g}}(\mathbf{x})-\mathbf{g}_{N T}(t, \mathbf{x})$.

Definition 2.1. We call $\bar{g}(\boldsymbol{x})$ the resonance term of $\boldsymbol{g}(t, \boldsymbol{x})$, denoted by $[\boldsymbol{g}(t, \boldsymbol{x})]_{R}, \boldsymbol{g}_{N}(t, \boldsymbol{x})$ the non-resonance term of $\boldsymbol{g}(t, \boldsymbol{x})$, denoted by $[\boldsymbol{g}(t, \boldsymbol{x})]_{N}$, and $\boldsymbol{g}_{N T}(t, \boldsymbol{x})$ the non-resonancetime term of $\boldsymbol{g}(t, \boldsymbol{x})$, denoted by $[\boldsymbol{g}(t, \boldsymbol{x})]_{N T}$.

Step 1. [Expansion of order $\varepsilon^{1}$ ] Expand $\mathbf{x}$ as

$$
\mathbf{x}=\mathbf{x}_{0}+\varepsilon \mathbf{x}_{1}+\mathcal{O}\left(\varepsilon^{2}\right) .
$$

Substitute (2.1) into Eq. (1.4), and equate the same degrees of $\varepsilon$, we get

$$
\begin{aligned}
& \dot{\mathbf{x}}_{0}=A \mathbf{x}_{0}, \\
& \dot{\mathbf{x}}_{1}=A \mathbf{x}_{1}+\mathbf{f}\left(\mathbf{x}_{0}\right),
\end{aligned}
$$

and we can easily solve them as

$$
\begin{aligned}
\mathbf{x}_{0} & =e^{A t} \mathbf{y}_{0}, \\
\mathbf{x}_{1} & =e^{A t} \mathbf{y}_{1}+e^{A t} \int_{0}^{t} e^{-A s} \mathbf{f}\left(e^{A s} \mathbf{y}_{0}\right) \mathrm{d} s \\
& =e^{A t} \mathbf{y}_{1}+e^{A t} \mathbf{R}^{(1)}\left(\mathbf{y}_{0}\right) t+e^{A t} \mathbf{F}^{(1)}\left(t, \mathbf{y}_{0}\right)-e^{A t} \mathbf{F}^{(1)}\left(0, \mathbf{y}_{0}\right),
\end{aligned}
$$

where $\mathbf{y}_{0}, \mathbf{y}_{1} \in \mathbb{C}^{n}$ are integral constants to be determined, and

$$
\begin{aligned}
& \mathbf{R}^{(1)}(\mathbf{y})=\left[e^{-A t} \mathbf{f}\left(e^{A t} \mathbf{y}\right)\right]_{R}=\sum_{i=1}^{n}\left(\sum_{\alpha \in N_{r}^{i}} \mathbf{f}_{\alpha}^{i} \mathbf{y}^{\alpha}\right) \mathbf{e}_{i}, \\
& \mathbf{F}^{(1)}(t, \mathbf{y})=\int^{t}\left[e^{-A s} \mathbf{f}\left(e^{A s} \mathbf{y}\right)\right]_{N} \mathrm{~d} s=\sum_{i=1}^{n}\left(\sum_{\alpha \notin N_{r}^{i}} \mathbf{f}_{\alpha}^{i} \frac{e^{\left(\langle\alpha, \Lambda\rangle-\lambda_{i}\right) t}}{\langle\alpha, \Lambda\rangle-\lambda_{i}} \mathbf{y}^{\alpha}\right) \mathbf{e}_{i}
\end{aligned}
$$


with $\left\{\mathbf{e}_{i}\right\}_{i=1}^{n}$ the eigenvectors of $A$, and

$$
N_{r}^{i}=\left\{\alpha \in \mathbb{N}^{n}:|\alpha| \geq 2,\langle\alpha, \Lambda\rangle \doteq \sum_{j=1}^{n} \lambda_{j} \alpha_{j}=\lambda_{i}\right\} .
$$

One can note that $\mathbf{R}^{(1)}(\mathbf{y})$ is the first order resonant term so that

$$
e^{-A t} \mathbf{R}^{(1)}\left(e^{A t} \mathbf{y}\right)=\mathbf{R}^{(1)}(\mathbf{y}), \quad t \in \mathbb{R},
$$

and

$$
\mathbf{F}^{(1)}(t, \mathbf{y})=\int^{t}\left[e^{-A s} \mathbf{f}\left(e^{A s} \mathbf{y}\right)\right]_{N} \mathrm{~d} s=\int^{t}\left(e^{-A s} \mathbf{f}\left(e^{A s} \mathbf{y}\right)-\mathbf{R}^{(1)}(\mathbf{y})\right) \mathrm{d} s .
$$

Then, we can get the naive expansion

$$
\begin{aligned}
\mathbf{x}= & e^{A t} \mathbf{y}_{0}+\varepsilon\left(e^{A t} \mathbf{y}_{1}+e^{A t} \mathbf{R}^{(1)}\left(\mathbf{y}_{0}\right) t+e^{A t} \mathbf{F}^{(1)}\left(t, \mathbf{y}_{0}\right)-e^{A t} \mathbf{F}^{(1)}\left(0, \mathbf{y}_{0}\right)\right)+\mathcal{O}\left(\varepsilon^{2}\right) \\
= & e^{A t}\left(\mathbf{y}_{0}+\varepsilon \mathbf{y}_{1}+\varepsilon \mathbf{R}^{(1)}\left(\mathbf{y}_{0}\right) \mu-\varepsilon \mathbf{F}^{(1)}\left(0, \mathbf{y}_{0}\right)\right)+\varepsilon e^{A t}\left(\mathbf{R}^{(1)}\left(\mathbf{y}_{0}\right)(t-\mu)+\mathbf{F}^{(1)}\left(t, \mathbf{y}_{0}\right)\right) \\
& +\mathcal{O}\left(\varepsilon^{2}\right)
\end{aligned}
$$

with a free parameter $\mu$ around time $t$. By renormalization as $\mathbf{y}=\mathbf{y}_{0}+\varepsilon \mathbf{y}_{1}+$ $\varepsilon \mathbf{R}^{(1)}\left(\mathbf{y}_{0}\right) \mu-\varepsilon \mathbf{F}^{(1)}\left(0, \mathbf{y}_{0}\right)$, the naive expansion can be formally rewritten as

$$
\mathbf{x}=e^{A t} \mathbf{y}+\varepsilon e^{A t}\left(\mathbf{R}^{(1)}(\mathbf{y})(t-\mu)+\mathbf{F}^{(1)}(t, \mathbf{y})\right)+\mathcal{O}\left(\varepsilon^{2}\right) .
$$

Step 2. [Expansion of order $\varepsilon^{2}$ ] Based on the first step, without loss of generality, we can represent $\mathbf{x}_{0}=e^{A t} \mathbf{y}$ and $\mathbf{x}_{1}=e^{A t}\left(\mathbf{R}^{(1)}(\mathbf{y})(t-\mu)+\mathbf{F}^{(1)}(t, \mathbf{y})\right)$, and expand $\mathbf{x}$ up to order $\varepsilon^{2}$ as

$$
\mathbf{x}=e^{A t} \mathbf{y}+\varepsilon e^{A t}\left(\mathbf{R}^{(1)}(\mathbf{y})(t-\mu)+\mathbf{F}^{(1)}(t, \mathbf{y})\right)+\varepsilon^{2} \mathbf{x}_{2}+\mathcal{O}\left(\varepsilon^{3}\right) .
$$

Now substitute (2.2) into (1.4), and equate the terms in $\varepsilon^{2}$, we have

$$
\dot{\mathbf{x}}_{2}=A \mathbf{x}_{2}+\frac{\partial}{\partial \mathbf{x}} \mathbf{f}\left(\mathbf{x}_{0}\right) \mathbf{x}_{1}
$$

and its general solution

$$
\begin{aligned}
\mathbf{x}_{2} & =e^{A t} \mathbf{y}_{2}+e^{A t} \int_{\mu}^{t} e^{-A s} \frac{\partial}{\partial \mathbf{x}} \mathbf{f}\left(e^{A s} \mathbf{y}\right) \mathbf{x}_{1} \mathrm{~d} s \\
& =e^{A t} \mathbf{y}_{2}+e^{A t} \mathbf{R}^{(2)}(\mathbf{y})(t-\mu)+e^{A t} \mathbf{F}^{(2)}(t, \mathbf{y})+e^{A t} \tilde{\mathbf{F}}^{(2)}(t-\mu, t, \mathbf{y})+e^{A t} \mathbf{F}^{(2)}(\mu, \mathbf{y}),
\end{aligned}
$$


where $\mathbf{y}_{2}$ is the integral constant to be determined, and

$$
\begin{gathered}
\mathbf{R}^{(2)}(\mathbf{y})=\left[e^{-A t} \frac{\partial}{\partial \mathbf{x}} \mathbf{f}\left(e^{A t} \mathbf{y}\right) \mathbf{x}_{1}(t)-\frac{\partial}{\partial \mathbf{y}} \mathbf{F}^{(1)}(t, \mathbf{y}) \mathbf{R}^{(1)}(\mathbf{y})\right]_{R}{ }^{\prime} \\
\mathbf{F}^{(2)}(t, \mathbf{y})=\int^{t}\left[e^{-A s} \frac{\partial}{\partial \mathbf{x}} \mathbf{f}\left(e^{A s} \mathbf{y}\right) \mathbf{x}_{1}(s)-\frac{\partial}{\partial \mathbf{y}} \mathbf{F}^{(1)}(s, \mathbf{y}) \mathbf{R}^{(1)}(\mathbf{y})\right]_{N} \mathrm{~d} s, \\
\tilde{\mathbf{F}}^{(2)}(t-\mu, t, \mathbf{y})=\int^{t}\left(\left[e^{-A s} \frac{\partial}{\partial \mathbf{x}} \mathbf{f}\left(e^{A s} \mathbf{y}\right) \mathbf{x}_{1}(s)\right]_{N T}+\frac{\partial}{\partial \mathbf{y}} \mathbf{F}^{(1)}(s, \mathbf{y}) \mathbf{R}^{(1)}(\mathbf{y})\right) \mathrm{d} s \\
=\int^{t}\left(\left[e^{-A s} \frac{\partial}{\partial \mathbf{y}} \mathbf{f}\left(e^{A s} \mathbf{y}\right)\right]_{R} \mathbf{R}^{(1)}(\mathbf{y})(s-\mu)\right. \\
\left.+\left[e^{-A s} \frac{\partial}{\partial \mathbf{y}} \mathbf{f}\left(e^{A s} \mathbf{y}\right)\right]_{N} \mathbf{R}^{(1)}(\mathbf{y})(s-\mu)+\frac{\partial}{\partial \mathbf{y}} \mathbf{F}^{(1)}(s, \mathbf{y}) \mathbf{R}^{(1)}(\mathbf{y})\right) \mathrm{d} s \\
=\frac{\partial}{\partial \mathbf{y}} \mathbf{F}^{(1)}(t, \mathbf{y}) \mathbf{R}^{(1)}(\mathbf{y})(t-\mu)+\frac{1}{2} \frac{\partial}{\partial \mathbf{y}} \mathbf{R}^{(1)}(\mathbf{y}) \mathbf{R}^{(1)}(\mathbf{y})(t-\mu)^{2} .
\end{gathered}
$$

Then make renormalization as $\mathbf{y}+\varepsilon^{2} \mathbf{y}_{2}+\varepsilon^{2} \mathbf{F}^{(2)}(\mu, \mathbf{y}) \rightarrow \mathbf{y}$, we can rewrite (2.2) as

$$
\begin{aligned}
\mathbf{x}= & e^{A t} \mathbf{y}+e^{A t}\left(\varepsilon \mathbf{R}^{(1)}(\mathbf{y})+\varepsilon^{2} \mathbf{R}^{(2)}(\mathbf{y})\right)(t-\mu) \\
& +e^{A t}\left(\varepsilon \mathbf{F}^{(1)}(t, \mathbf{y})+\varepsilon^{2} \mathbf{F}^{(2)}(t, \mathbf{y})\right) \\
& +\varepsilon^{2} e^{A t} \tilde{\mathbf{F}}^{(2)}(t-\mu, t, \mathbf{y})+\mathcal{O}\left(\varepsilon^{3}\right) .
\end{aligned}
$$

Step 3. [Expansion of order $\varepsilon^{m+1}$ ] Assume we can expand $\mathbf{x}$ up to order $\varepsilon^{m}$ as

$$
\begin{aligned}
\mathbf{x}= & e^{A t} \mathbf{y}+e^{A t} \sum_{i=1}^{m} \varepsilon^{i} \mathbf{R}^{(i)}(\mathbf{y})(t-\mu)+e^{A t} \sum_{i=1}^{m} \varepsilon^{i} \mathbf{F}^{(i)}(t, \mathbf{y}) \\
& +e^{A t} \sum_{i=2}^{m} \varepsilon^{i} \tilde{\mathbf{F}}^{(i)}(t-\mu, t, \mathbf{y})+\mathcal{O}\left(\varepsilon^{m+1}\right)
\end{aligned}
$$

with

$$
\tilde{\mathbf{F}}^{(i)}(t-\mu, t, \mathbf{y})=\sum_{j=1}^{i-1} \frac{\partial}{\partial \mathbf{y}} \mathbf{F}^{(j)}(t, \mathbf{y}) \mathbf{R}^{(i-j)}(\mathbf{y})(t-\mu)+\mathcal{O}\left((t-\mu)^{2}\right), \quad i=2, \ldots, m .
$$

Let

$$
\mathbf{x}_{i}=e^{A t} \mathbf{R}^{(i)}(\mathbf{y})(t-\mu)+e^{A t} \mathbf{F}^{(i)}(t, \mathbf{y})+e^{A t} \tilde{\mathbf{F}}^{(i)}(t-\mu, t, \mathbf{y}), \quad i=2, \ldots, m,
$$


and expand $\mathbf{x}$ up to order $\varepsilon^{m+1}$ as

$$
\begin{aligned}
\mathbf{x}= & e^{A t} \mathbf{y}+e^{A t} \sum_{i=1}^{m} \varepsilon^{i} \mathbf{R}^{(i)}(\mathbf{y})(t-\mu)+e^{A t} \sum_{i=1}^{m} \varepsilon^{i} \mathbf{F}^{(i)}(t, \mathbf{y}) \\
& +\sum_{i=2}^{m} \varepsilon^{i} e^{A t} \tilde{\mathbf{F}}^{(i)}(t-\mu, t, \mathbf{y})+\varepsilon^{m+1} \mathbf{x}_{m+1}+\mathcal{O}\left(\varepsilon^{m+2}\right) .
\end{aligned}
$$

Substitute (2.5) in (1.4), and equate the terms of $\varepsilon^{m+1}$, we get

$$
\begin{aligned}
\dot{\mathbf{x}}_{m+1} & =A \mathbf{x}_{m+1}+\left.\frac{\mathrm{d}^{m}}{\mathrm{~d} \varepsilon^{m}} \mathbf{f}\left(\sum_{l=0}^{m} \varepsilon^{l} \mathbf{x}_{l}(t)+\mathcal{O}\left(\varepsilon^{m+1}\right)\right)\right|_{\varepsilon=0} \\
& =A \mathbf{x}_{m+1}+\sum_{j=1}^{m} \frac{1}{j !} \partial^{j} \mathbf{f}\left(\mathbf{x}_{0}\right) \sum_{\substack{k_{1}+\cdots+k_{j}=m \\
k_{i} \geq 1}} \mathbf{x}_{k_{1}} \cdots \mathbf{x}_{k_{j}},
\end{aligned}
$$

where $\partial^{j} \mathbf{f}$, the $j$-th derivative of $\mathbf{f}$, is a tensor with $j$ indices ( $j$ must be contracted with the vectors $\mathbf{x}_{k_{1}}, \cdots, \mathbf{x}_{k_{j}}$ ). Eq. (2.6) can be solved as

$$
\begin{aligned}
\mathbf{x}_{m+1}= & e^{A t} \mathbf{y}_{m+1}+e^{A t} \int_{\mu}^{t} e^{-A s} \sum_{j=1}^{m} \frac{1}{j !} \partial^{j} \mathbf{f}\left(e^{A s} \mathbf{y}\right) \sum_{\substack{k_{1}+\cdots+k_{j}=m \\
k_{i} \geq 1}} \mathbf{x}_{k_{1}} \cdots \mathbf{x}_{k_{j}} \mathrm{~d} s \\
= & e^{A t} \mathbf{y}_{m+1}+e^{A t} \mathbf{R}^{(m+1)}(\mathbf{y})(t-\mu)+e^{A t} \mathbf{F}^{(m+1)}(t, \mathbf{y}) \\
& +e^{A t} \tilde{\mathbf{F}}^{(m+1)}(t-\mu, t, \mathbf{y})+e^{A t} \mathbf{F}^{(m+1)}(\mu, \mathbf{y}),
\end{aligned}
$$

where $\mathbf{y}_{m+1}$ is the integral constant to be determined, and

$$
\begin{aligned}
& \mathbf{R}^{(m+1)}(\mathbf{y}) \\
= & {\left[e^{-A t} \sum_{j=1}^{m} \frac{1}{j !} \partial^{j} \mathbf{f}\left(\mathbf{x}_{0}\right) \sum_{\substack{k_{1}+\cdots+k_{j}=m \\
k_{i} \geq 1}} \mathbf{x}_{k_{1}} \cdots \mathbf{x}_{k_{j}}-\sum_{j=1}^{m} \frac{\partial}{\partial \mathbf{y}} \mathbf{F}^{(j)}(t, \mathbf{y}) \mathbf{R}^{(m+1-j)}(\mathbf{y})\right]_{R}, } \\
& \mathbf{F}^{(m+1)}(t, \mathbf{y}) \\
= & \int^{t}\left[e^{-A s} \sum_{j=1}^{m} \frac{1}{j !} \partial^{j} \mathbf{f}\left(\mathbf{x}_{0}\right) \sum_{\substack{k_{1}+\cdots+k_{j}=m \\
k_{i} \geq 1}} \mathbf{x}_{k_{1}} \cdots \mathbf{x}_{k_{j}}-\sum_{j=1}^{m} \frac{\partial}{\partial \mathbf{y}} \mathbf{F}^{(j)}(s, \mathbf{y}) \mathbf{R}^{(m+1-j)}(\mathbf{y})\right]_{N} \mathrm{~d} s,
\end{aligned}
$$




$$
\begin{aligned}
& \tilde{\mathbf{F}}^{(m+1)}(t-\mu, t, \mathbf{y}) \\
= & \int^{t}\left(\left[e^{-A s} \sum_{j=1}^{m} \frac{1}{j !} \partial^{j} \mathbf{f}\left(\mathbf{x}_{0}\right) \sum_{\substack{k_{1}+\cdots+k_{j}=m \\
k_{i} \geq 1}} \mathbf{x}_{k_{1}} \cdots \mathbf{x}_{k_{j}}\right]_{N T}+\sum_{j=1}^{m} \frac{\partial}{\partial \mathbf{y}} \mathbf{F}^{(j)}(s, \mathbf{y}) \mathbf{R}^{(m+1-j)}(\mathbf{y})\right) \mathrm{d} s \\
= & \sum_{j=1}^{m} \frac{\partial}{\partial \mathbf{y}} \mathbf{F}^{(j)}(t, \mathbf{y}) \mathbf{R}^{(m+1-j)}(\mathbf{y})(t-\mu)+\mathcal{O}\left((t-\mu)^{2}\right) .
\end{aligned}
$$

Make renormalization as $\mathbf{y}+\varepsilon^{m+1} \mathbf{y}_{m+1}+\varepsilon^{m+1} \mathbf{F}^{(m+1)}(\mu, \mathbf{y}) \rightarrow \mathbf{y}$, we can rewrite (2.5) as

$$
\begin{aligned}
\mathbf{x}= & e^{A t} \mathbf{y}+e^{A t} \sum_{i=1}^{m+1} \varepsilon^{i} \mathbf{R}^{(i)}(\mathbf{y})(t-\mu)+e^{A t} \sum_{i=1}^{m+1} \varepsilon^{i} \mathbf{F}^{(i)}(t, \mathbf{y}) \\
& +e^{A t} \sum_{i=2}^{m} \varepsilon^{i} \tilde{\mathbf{F}}^{(i)}(t-\mu, t, \mathbf{y})+\mathcal{O}\left(\varepsilon^{m+2}\right)
\end{aligned}
$$

Step 4. [Approximate solution] By noting that $\mathbf{x}(t)$ should be independent of the parameter $\mu$, we have

$$
\left.\frac{\mathrm{d} \mathbf{x}}{\mathrm{d} \mu}\right|_{\mu=t}=0
$$

Taking it into (2.4), we obtain

$$
\begin{aligned}
\mathcal{O}\left(\varepsilon^{m+1}\right)= & e^{A t} \frac{\mathrm{d} \mathbf{y}}{\mathrm{d} t}-e^{A t} \sum_{i=1}^{m} \varepsilon^{i} \mathbf{R}^{(i)}(\mathbf{y})+e^{A t} \sum_{i=1}^{m} \varepsilon^{i} \frac{\partial}{\partial \mathbf{y}} \mathbf{F}^{(i)}(t, \mathbf{y}) \frac{\mathrm{d} \mathbf{y}}{\mathrm{d} t} \\
& -\sum_{i=2}^{m} \varepsilon^{i} e^{A t} \sum_{j=1}^{i-1} \frac{\partial}{\partial \mathbf{y}} \mathbf{F}^{(j)}(t, \mathbf{y}) \mathbf{R}^{(i-j)}(\mathbf{y}) \\
= & e^{A t}\left(i d+\sum_{i=1}^{m} \varepsilon^{i} \frac{\partial}{\partial \mathbf{y}} \mathbf{F}^{(i)}(t, \mathbf{y})\right) \frac{\mathrm{d} \mathbf{y}}{\mathrm{d} t}-e^{A t} \sum_{i=1}^{m} \varepsilon^{i} \mathbf{R}^{(i)}(\mathbf{y}) \\
& -e^{A t}\left(\sum_{i=1}^{m} \varepsilon^{i} \frac{\partial}{\partial \mathbf{y}} \mathbf{F}^{(j)}(t, \mathbf{y})\right)\left(\sum_{j=1}^{m} \varepsilon^{j} \mathbf{R}^{(j)}(\mathbf{y})\right) \\
= & e^{A t}\left(i d+\sum_{i=1}^{m} \varepsilon^{i} \frac{\partial}{\partial \mathbf{y}} \mathbf{F}^{(i)}(t, \mathbf{y})\right) \frac{\mathrm{d} \mathbf{y}}{\mathrm{d} t} \\
& -e^{A t}\left(i d+\sum_{i=1}^{m} \varepsilon^{i} \frac{\partial}{\partial \mathbf{y}} \mathbf{F}^{(i)}(t, \mathbf{y})\right)\left(\sum_{j=1}^{m} \varepsilon^{j} \mathbf{R}^{(j)}(\mathbf{y})\right)
\end{aligned}
$$




$$
=e^{A t}\left(i d+\sum_{i=1}^{m} \varepsilon^{i} \frac{\partial}{\partial \mathbf{y}} \mathbf{F}^{(i)}(t, \mathbf{y})\right)\left(\frac{\mathrm{d} \mathbf{y}}{\mathrm{d} t}-\sum_{j=1}^{m+1} \varepsilon^{j} \mathbf{R}^{(j)}(\mathbf{y})\right) .
$$

Then we get the $m$-th RG equation

$$
\frac{\mathrm{d} \mathbf{y}}{\mathrm{d} t}=\sum_{j=1}^{m} \varepsilon^{j} \mathbf{R}^{(j)}(\mathbf{y})
$$

and the $m$-th order approximate solution

$$
\mathbf{x}_{\varepsilon}=e^{A t} \mathbf{y}+e^{A t} \sum_{i=1}^{m} \varepsilon^{i} \mathbf{F}^{(i)}(t, \mathbf{y}) .
$$

It is easy to conclude that $\mathbf{x}_{\varepsilon}(t)$ satisfies

$$
\begin{aligned}
\dot{\mathbf{x}}_{\varepsilon}= & A \mathbf{x}_{\varepsilon}+e^{A t} \dot{\mathbf{y}}+e^{A t} \sum_{i=1}^{m} \varepsilon^{i} \frac{\mathrm{d}}{\mathrm{d} t} \mathbf{F}^{(i)}(t, \mathbf{y}) \\
= & A \mathbf{x}_{\varepsilon}+e^{A t} \sum_{j=1}^{m} \varepsilon^{j} \mathbf{R}^{(j)}(\mathbf{y})+e^{A t} \sum_{i=1}^{m} \varepsilon^{i} \frac{\partial}{\partial t} \mathbf{F}^{(i)}(t, \mathbf{y}) \\
& +e^{A t} \sum_{i=1}^{m} \varepsilon^{i} \frac{\partial}{\partial \mathbf{y}} \mathbf{F}^{(i)}(t, \mathbf{y}) \sum_{j=1}^{m} \varepsilon^{j} \mathbf{R}^{(j)}(\mathbf{y}) \\
= & A \mathbf{x}_{\varepsilon}+\varepsilon \mathbf{f}\left(\mathbf{x}_{\varepsilon}\right)+\varepsilon^{m+1} \mathbf{S}_{m}(t, \mathbf{y}, \varepsilon)
\end{aligned}
$$

where

$$
\begin{aligned}
\mathbf{S}_{m}(t, \mathbf{y}, \varepsilon) & =\sum_{l=0}^{m-1}\left(\left.\frac{\mathrm{d}^{l}}{\mathrm{~d} \varepsilon^{l}} \mathbf{f}\left(\mathbf{x}_{\varepsilon}(t)\right)\right|_{\varepsilon=0}\right) \varepsilon^{l+1}-\varepsilon \mathbf{f}\left(\mathbf{x}_{\varepsilon}(t)\right) \\
& =\sum_{l=m}^{+\infty}\left(\left.\frac{\mathrm{d}^{l}}{\mathrm{~d} \varepsilon^{l}} \mathbf{f}\left(\mathbf{x}_{\varepsilon}(t)\right)\right|_{\varepsilon=0}\right) \varepsilon^{l+1}
\end{aligned}
$$

Remark 2.1. (1) Compared with the RG procedures in Temam [20] and Chiba [3], our strategy is more direct and much closer to the original RG idea in [2].

(2) By induction, one can easily conclude that $\mathbf{S}_{m}(t, \mathbf{y}, \varepsilon)=\mathcal{O}\left(\|\mathbf{y}\|^{m}\right)$, this, combined with Definition 2.1, promotes us to investigate the normal form of the original equations later.

(3) The above strategy can be extended to more general perturbation $\mathbf{f}(\mathbf{x}, t, \varepsilon)$, which is polynomial in $\mathbf{x}$, almost periodic in $t$ and $C^{r}$ or analytic in $\varepsilon$. 


\section{Convergence estimates}

In order to investigate the error estimates between the original solution and approximate solution, we make the following assumption.

(H) The solution of the initial problem

$$
\left\{\begin{array}{l}
\frac{\mathrm{d} \mathbf{y}}{\mathrm{d} \tau}=\sum_{j=1}^{m} \varepsilon^{j-1} \mathbf{R}^{(j)}(\mathbf{y}), \\
\mathbf{y}(0)=\mathbf{y}_{0}
\end{array}\right.
$$

exists on interval $(a, b)$ with $a<0<b$ independent of $\varepsilon$.

Theorem 3.1. Assume that $(\mathbf{H})$ holds and $A$ is a diagonalizable matrix with all the eigenvalues having real parts not more than zero. Let $\boldsymbol{x}(t)$ be the solution of (1.4) with initial condition $\boldsymbol{x}(0)=\boldsymbol{x}_{0}$, and $\left\|\boldsymbol{y}_{0}-\boldsymbol{x}_{0}\right\|=\mathcal{O}\left(\varepsilon^{m}\right)$ for some $m \in \mathbb{N}$. Then, for any $T \in(0, b)$, there exists $\varepsilon_{0}>0$, such that for any $\varepsilon \in\left(0, \varepsilon_{0}\right)$

$$
\left|x(t)-x_{\varepsilon}(t)\right|<M \varepsilon^{m}, \quad t \in[0, T / \varepsilon],
$$

where $M>0$ is dependent on $T, m, \varepsilon_{0}$ and $\boldsymbol{y}_{0}$.

Proof. Let $\mathbf{w}_{\varepsilon}(t)=\mathbf{x}(t)-\mathbf{x}_{\varepsilon}(t)$. Then by (2.11), we have

$$
\begin{aligned}
& \dot{\mathbf{w}}_{\varepsilon}=A \mathbf{w}_{\varepsilon}+\varepsilon\left(\mathbf{f}(\mathbf{x})-\mathbf{f}\left(\mathbf{x}_{\varepsilon}\right)\right)-\varepsilon^{m+1} \mathbf{S}_{m}(t, \mathbf{y}, \varepsilon), \\
& \mathbf{w}_{\varepsilon}(t)= e^{A t} \mathbf{w}_{\varepsilon}(0)+\varepsilon \int_{0}^{t} e^{A(t-s)}\left(\mathbf{f}(\mathbf{x}(s))-\mathbf{f}\left(\mathbf{x}_{\varepsilon}(s)\right)\right) \mathrm{d} s \\
&-\varepsilon^{m+1} \int_{0}^{t} e^{A(t-s)} \mathbf{S}_{m}(s, \mathbf{y}(s), \varepsilon) \mathrm{d} s .
\end{aligned}
$$

Therefore

$$
\begin{aligned}
\left\|\mathbf{w}_{\varepsilon}(t)\right\| \leq & \left\|\mathbf{w}_{\mathcal{\varepsilon}}(0)\right\|+\varepsilon \int_{0}^{t}\left\|\left(\mathbf{f}(\mathbf{x}(s))-\mathbf{f}\left(\mathbf{x}_{\mathcal{\varepsilon}}(s)\right)\right)\right\| \mathrm{d} s \\
& +\varepsilon^{m+1} \int_{0}^{t}\left\|\mathbf{S}_{m}(s, \mathbf{y}(s), \varepsilon)\right\| \mathrm{d} s .
\end{aligned}
$$

By $(\mathbf{H})$, there exists a positive constant $C_{0}$ which only depends on $T$ and $\mathbf{y}_{0}$, such that the solution $\mathbf{y}(t)$ of (2.9) with $\mathbf{y}(0)=\mathbf{y}_{0}$ can be bounded as

$$
\|\mathbf{y}(t)\| \leq C_{0}, \quad t \in[0, T / \varepsilon]
$$


Hence, there exists a positive constant $C_{1}$ which depends on $T$ and $\mathbf{y}_{0}$, such that for $t \in\left[0, \frac{T}{\varepsilon}\right]$,

$$
\begin{aligned}
& \left\|\mathbf{R}^{(j)}(\mathbf{y}(t))\right\| \leq C_{1}, \quad j=1, \ldots, m, \\
& \left\|e^{A t} \mathbf{F}^{(1)}(t, \mathbf{y}(t))\right\| \leq C_{1}, \\
& \left\|\frac{\partial}{\partial \mathbf{y}} e^{A t} \mathbf{F}^{(1)}(t, \mathbf{y}(t))\right\| \leq C_{1} .
\end{aligned}
$$

Now, it is not difficult to see that both $e^{A t} \mathbf{F}^{(1)}(t, \mathbf{y}(t))$ and $\frac{\partial}{\partial \mathbf{y}} e^{A t} \mathbf{F}^{(1)}(t, \mathbf{y}(t))$ only contain the terms which are exponential in $t$ and the corresponding exponents are non-positive. By induction, we assume that, for $j \in\{1, \ldots, k\}, e^{A t} \mathbf{F}^{(j)}(t, \mathbf{y}(t))$ and $\frac{\partial}{\partial \mathbf{y}} e^{A t} \mathbf{F}^{(j)}(t, \mathbf{y}(t))$ only contain the terms which are exponential in $t$ and the corresponding exponents are non-positive. Then

$$
\begin{array}{r}
e^{A t} \mathbf{F}^{(k+1)}(t, \mathbf{y})=e^{A t} \int^{t}\left[e ^ { - A s } \left(\sum_{j=1}^{m} \frac{1}{j !} \partial^{j} \mathbf{f}\left(\mathbf{x}_{0}\right) \sum_{\substack{k_{1}+\cdots+k_{j}=m \\
k_{i} \geq 1}} \mathbf{x}_{k_{1}} \cdots \mathbf{x}_{k_{j}}\right.\right. \\
\left.\left.-\sum_{j=1}^{m} \frac{\partial}{\partial \mathbf{y}} e^{A s} \mathbf{F}^{(j)}(s, \mathbf{y}) \mathbf{R}^{(m+1-j)}(\mathbf{y})\right)\right]_{N} \mathrm{~d} s,
\end{array}
$$

which implies that $e^{A t} \mathbf{F}^{(k+1)}(t, \mathbf{y})$ only contains all the terms exponential in $t$ with non-positive exponents, and therefore, there exists a positive constant $C_{2}$ which depends on $T, m$ and $\mathbf{y}_{0}$, such that for any $j \in\{1, \ldots, m\}$ and $t \in[0, T / \varepsilon]$,

$$
\begin{aligned}
& \left\|e^{A t} \mathbf{F}^{(j)}(t, \mathbf{y}(t))\right\| \leq C_{2} \\
& \left\|\frac{\partial}{\partial \mathbf{y}} e^{A t} \mathbf{F}^{(j)}(t, \mathbf{y}(t))\right\| \leq C_{2}
\end{aligned}
$$

These inequalities imply that there exist a sufficiently small $\varepsilon_{0}>0$ and a positive constant $C_{3}$ which depends on $T, m, \varepsilon_{0}$ and $\mathbf{y}_{0}$, such that for $\varepsilon \in\left(0, \varepsilon_{0}\right)$ and $t \in$ $[0, T / \varepsilon]$,

$$
\left\|\mathbf{x}_{\varepsilon}(t)\right\| \leq C_{3}, \quad\left\|\mathbf{S}_{m}(t, \mathbf{y}, \varepsilon)\right\| \leq C_{3} .
$$

Now, for given $T \in(0, b)$ and $\varepsilon \in\left(0, \varepsilon_{0}\right)$, let

$$
t_{0}=\sup \left\{t_{1} \in[0, T / \varepsilon] \mid\left\|\mathbf{w}_{\varepsilon}(t)\right\| \leq 1, t \in\left[0, t_{1}\right]\right\} .
$$


Obviously, $t_{0}>0$ for sufficient small $\varepsilon_{0}$, and there exists a positive constant $C_{4}$ which depends on $C_{3}$ such that

$$
\left\|\mathbf{w}_{\varepsilon}(t)\right\| \leq\left\|\mathbf{w}_{\varepsilon}(0)\right\|+\varepsilon C_{4} \int_{0}^{t}\left\|\mathbf{w}_{\varepsilon}(s)\right\| \mathrm{d} s+C_{3} T \varepsilon^{m}, \quad t \in\left[0, t_{0}\right]
$$

Hence, by Gronwall's inequality,

$$
\left\|\mathbf{w}_{\mathcal{\varepsilon}}(t)\right\| \leq\left(\left\|\mathbf{w}_{\mathcal{\varepsilon}}(0)\right\|+C_{3} T \varepsilon^{m}\right) e^{C_{4} T} \leq M \varepsilon^{m}, \quad t \in\left[0, t_{0}\right],
$$

where $M$ is a positive constant which depends on $T, m$ and $\mathbf{y}_{0}$. Therefore, with sufficient small $\varepsilon_{0}$, one can immediately conclude that $t_{0}=T / \varepsilon$.

Corollary 3.1. Assume that $(\mathbf{H})$ holds and $A$ is a diagonalizable matrix with all the eigenvalues having negative real parts. Let $x(t)$ be the solution of (1.4) with initial condition $\boldsymbol{x}(0)=\boldsymbol{x}_{0}$, and $\left\|\boldsymbol{y}_{0}-\boldsymbol{x}_{0}\right\|=\mathcal{O}\left(\varepsilon^{m}\right)$ for some $m \in \mathbb{N}$. Then, for any $T \in(0, b)$, there exist $\varepsilon_{0}>0$ and $\sigma<\alpha$, such that, for any $\varepsilon \in\left(0, \varepsilon_{0}\right)$,

$$
\left\|x(t)-x_{\varepsilon}(t)\right\|<M \varepsilon^{m} e^{-\sigma t}, \quad t \in[0, T / \varepsilon],
$$

where $M$ is a positive constant which depends on $T, m, \varepsilon_{0}$ and $\boldsymbol{y}_{0}$. Moreover, if the solution of (3.1) is globally bounded, then (3.4) is valid for $t \in(0,+\infty)$.

Proof. Let $\lambda_{1}, \cdots, \lambda_{n}$ be eigenvalues of $A$, then there exists a positive constant $\alpha$ such that $\operatorname{Re} \lambda_{i}<-\alpha, i=1, \ldots, n$. Therefore, there exists a positive constant $C_{0}$ which depends on $\alpha$, such that

$$
\begin{aligned}
\left\|\mathbf{w}_{\varepsilon}(t)\right\| \leq & C_{0} e^{-\alpha t}\left\|\mathbf{w}_{\varepsilon}(0)\right\|+\varepsilon C_{0} \int_{0}^{t} e^{-\alpha(t-s)}\left\|\left(\mathbf{f}(\mathbf{x}(s))-\mathbf{f}\left(\mathbf{x}_{\varepsilon}(s)\right)\right)\right\| \mathrm{d} s \\
& +\varepsilon^{m+1} C_{0} \int_{0}^{t} e^{-\alpha(t-s)}\left\|\mathbf{S}_{m}(s, \mathbf{y}(s), \varepsilon)\right\| \mathrm{d} s .
\end{aligned}
$$

From the proof of Theorem 3.1, there exists a positive constant $C_{1}$ which depends on $T$ and $\mathbf{y}_{0}$, such that, the solution $\mathbf{y}(t)$ of (2.9) with $\mathbf{y}(0)=\mathbf{y}_{0}$ can be bounded as

$$
\begin{array}{ll}
\|\mathbf{y}(t)\| \leq C_{1}, & t \in[0, T / \varepsilon], \\
\left\|\mathbf{R}^{(j)}(\mathbf{y}(t))\right\| \leq C_{1}, \quad t \in[0, T / \varepsilon], \quad j \in\{1, \ldots, m\} .
\end{array}
$$

Furthermore, for any $j \in\{1, \ldots, m\}, e^{A t} \mathbf{F}^{(j)}(t, \mathbf{y}(t))$ and $\frac{\partial}{\partial \mathbf{y}} e^{A t} \mathbf{F}^{(j)}(t, \mathbf{y}(t))$ only contain the terms which are exponential in $t$ with non-positive real part exponents. 
We note that the terms in $e^{A t} \mathbf{F}^{(j)}(t, \mathbf{y}(t))$ and $\frac{\partial}{\partial \mathbf{y}} e^{A t} \mathbf{F}^{(j)}(t, \mathbf{y}(t))$ are, in fact, exponential in $t$ with negative real part exponents, that is to say, there exist $\beta \geq \alpha$ and a positive constant $C_{2}$ which depends on $T, m$ and $\mathbf{y}_{0}$, such that for any $j \in\{1, \ldots, m\}$ and $t \in[0, T / \varepsilon]$,

$$
\begin{aligned}
& \left\|\mathbf{R}^{(j)}(\mathbf{y}(t))\right\| \leq C_{2}, \\
& \left\|e^{A t} \mathbf{F}^{(j)}(t, \mathbf{y}(t))\right\| \leq C_{2} e^{-\beta t}, \\
& \left\|e^{A t} \frac{\partial}{\partial \mathbf{y}} \mathbf{F}^{(j)}(t, \mathbf{y}(t))\right\| \leq C_{2} e^{-\beta t}, \\
& \left\|\mathbf{x}_{j}(t)\right\| \leq C_{2} e^{-\beta t} .
\end{aligned}
$$

These inequalities imply that there exist a sufficiently small $\varepsilon_{0}>0$ and a positive constant $C_{3}$ which depends on $T, m, \varepsilon_{0}$ and $\mathbf{y}_{0}$, such that for any $\varepsilon \in\left(0, \varepsilon_{0}\right)$ and $t \in[0, T / \varepsilon]$,

$$
\left\|\mathbf{x}_{\varepsilon}(t)\right\| \leq C_{3} e^{-\alpha t}, \quad\left\|\mathbf{S}_{m}(t, \mathbf{y}, \varepsilon)\right\| \leq C_{3} e^{-\alpha t} .
$$

For given $T \in(0, b)$ and $\varepsilon \in\left(0, \varepsilon_{0}\right)$, let

$$
t_{0}=\sup \left\{t_{1} \in[0, T / \varepsilon] \mid\left\|\mathbf{w}_{\varepsilon}(t)\right\| \leq 1, t \in\left[0, t_{1}\right]\right\} .
$$

With the same idea in Theorem 3.1, we can find that $t_{0}>0$ for sufficient small $\varepsilon_{0}$, and there exists a positive constant $C_{5}$ which depends on $C_{3}$ such that

$$
e^{\alpha t}\left\|\mathbf{w}_{\mathcal{\varepsilon}}(t)\right\| \leq\left\|C_{0} \mathbf{w}_{\mathcal{\varepsilon}}(0)\right\|+\varepsilon C_{5} \int_{0}^{t} e^{\alpha s}\left\|\mathbf{w}_{\mathcal{E}}(s)\right\| \mathrm{d} s+C_{5} \varepsilon^{m+1} t, \quad t \in\left[0, t_{0}\right] .
$$

Hence, by Gronwall's inequality,

$$
\left\|\mathbf{w}_{\mathcal{\varepsilon}}(t)\right\| \leq\left(C_{0}\left\|\mathbf{w}_{\mathcal{\varepsilon}}(0)\right\|+C_{6} \varepsilon^{m}\right) e^{-\sigma t} \leq M e^{-\sigma t} \varepsilon^{m}, \quad t \in\left[0, t_{0}\right],
$$

here $\sigma \leq \alpha-\varepsilon_{0} C_{5}$. Note that $C_{3}, C_{5}, C_{6}$ and $M$ are polynomially dependent on $\varepsilon_{0}$, so $\sigma$ can be chosen of order $\mathcal{O}(1)$. Furthermore, since $M$ is a positive constant which depends on $T, m, \varepsilon_{0}$ and $\mathbf{y}_{0}$, with sufficient small $\varepsilon_{0}$, we can conclude that $t_{0}=T / \varepsilon$. Then, the whole proof can be completed by noting the fact that all the constants obtained above will be independent of $T$, if the solution of (3.1) is globally bounded.

Corollary 3.2. Assume that $(\mathbf{H})$ holds and $A$ is a diagonalizable matrix with all the eigenvalues having zero real parts. Let $\boldsymbol{x}(t)$ be the solution of (1.4) with initial condition 
$\boldsymbol{x}(0)=x_{0}$, and $\left\|\boldsymbol{y}_{0}-\boldsymbol{x}_{0}\right\|=\mathcal{O}\left(\varepsilon^{m}\right)$ for some $m \in \mathbb{N}$. Then, for any $\left[T_{1}, T_{2}\right] \subset(a, b)$ with $T_{1} \leq 0 \leq T_{2}$, there exist $\varepsilon_{0}>0$, such that for any $\varepsilon \in\left(0, \varepsilon_{0}\right)$,

$$
\left\|x(t)-x_{\varepsilon}(t)\right\|<M \varepsilon^{m}, \quad t \in\left[T_{1} / \varepsilon, T_{2} / \varepsilon\right],
$$

where $M>0$ is a positive constant which depends on $T_{1}, T_{2}, m, \varepsilon_{0}$ and $\boldsymbol{y}_{0}$.

Proof. The statement can be proved similar to the proof of Theorem 3.1.

We remark that a same result as Corollary 3.2 was proved by Chiba [5] by combining the RG method and the KBM idea [18].

\section{Normal form}

As referred in Remark 2.1, we make a further investigation on some properties related to the normal form.

Lemma 4.1. For any $j \in \mathbb{N}, \mu \in \mathbb{R}$ and $\boldsymbol{y} \in \mathbb{C}^{n}$,

$$
\begin{aligned}
& \boldsymbol{R}^{(j)}\left(e^{A \mu} \boldsymbol{y}\right)=e^{A \mu} \boldsymbol{R}^{(j)}(\boldsymbol{y}), \\
& e^{A t} \boldsymbol{F}^{(j)}\left(t, e^{A \mu} \boldsymbol{y}\right)=e^{A(t+\mu)} \boldsymbol{F}^{(j)}(t+\mu, \boldsymbol{y}) .
\end{aligned}
$$

Proof. Firstly, for $j=1$,

$$
\begin{aligned}
& \mathbf{R}^{(1)}\left(e^{A \mu} \mathbf{y}\right)=\left[e^{-A t} \mathbf{f}\left(e^{A(t+\mu)} \mathbf{y}\right)\right]_{R}=e^{A \mu}\left[e^{-A(t+\mu)} \mathbf{f}\left(e^{A(t+\mu)} \mathbf{y}\right)\right]_{R}=e^{A \mu} \mathbf{R}^{(1)}(\mathbf{y}), \\
& e^{A t} \mathbf{F}^{(1)}\left(t, e^{A \mu} \mathbf{y}\right)=e^{A t} \int^{t}\left(e^{-A s} \mathbf{f}\left(e^{A(s+\mu)} \mathbf{y}\right)-e^{A \mu} \mathbf{R}^{(1)}(\mathbf{y})\right) \mathrm{d} s \\
&=e^{A(t+\mu)} \int^{t}\left(e^{-A(s+\mu)} \mathbf{f}\left(e^{A(s+\mu)} \mathbf{y}\right)-\mathbf{R}^{(1)}(\mathbf{y})\right) \mathrm{d} s \\
&=e^{A(t+\mu)} \int^{t+\mu}\left(e^{-A s} \mathbf{f}\left(e^{A s} \mathbf{y}\right)-\mathbf{R}^{(1)}(\mathbf{y})\right) \mathrm{d} s \\
&=e^{A(t+\mu)} \mathbf{F}^{(1)}(t+\mu, \mathbf{y}) .
\end{aligned}
$$

Now, by inductions, we assume that (4.1) and (4.2) hold for all $j \in\{1, \ldots, m\}$, then for any $j \in\{1, \ldots, m\}, \mu \in \mathbb{R}$ and $\mathbf{z} \in \mathbb{C}^{n}$,

$$
\begin{aligned}
& \frac{\partial}{\partial \mathbf{y}} \mathbf{F}^{(j)}\left(t, e^{A \mu} \mathbf{z}\right)=e^{-A t} \frac{\partial}{\partial \mathbf{z}} e^{A t} \mathbf{F}^{(j)}\left(t, e^{A \mu} \mathbf{z}\right) e^{-A \mu} \\
&=e^{-A t} \frac{\partial}{\partial \mathbf{z}} e^{A(t+\mu)} \mathbf{F}^{(j)}(t+\mu, \mathbf{z}) e^{-A \mu}, \\
& \mathbf{x}_{j}\left(t, e^{A \mu} \mathbf{z}\right)=\mathbf{x}_{j}(t+\mu, \mathbf{z}) .
\end{aligned}
$$


Hence

$$
\begin{aligned}
& \mathbf{R}^{(m+1)}\left(e^{A \mu} \mathbf{y}\right) \\
&= {\left[e^{-A t} \sum_{j=1}^{m} \frac{1}{j !} \partial^{j} \mathbf{f}\left(\mathbf{x}_{0}(t+\mu)\right) \sum_{\substack{k_{1}+\cdots+k_{j}=m \\
k_{i} \geq 1}} \mathbf{x}_{k_{1}}(t+\mu) \cdots \mathbf{x}_{k_{j}}(t+\mu)\right.} \\
&\left.-e^{-A t} \sum_{j=1}^{m} \frac{\partial}{\partial \mathbf{z}} e^{A(t+\mu)} \mathbf{F}^{(j)}(t+\mu, \mathbf{z}) \mathbf{R}^{(m+1-j)}(\mathbf{y})\right]_{R}=e^{A \mu} \mathbf{R}^{(m+1)}(\mathbf{y}), \\
& e^{A t} \mathbf{F}^{(m+1)}\left(t, e^{A \mu} \mathbf{y}\right) \\
&= e^{A t} \int^{t}\left[e^{-A s} \sum_{j=1}^{m} \frac{1}{j !} \partial^{j} \mathbf{f}\left(\mathbf{x}_{0}(s+\mu)\right) \sum_{k_{1}+\cdots+k_{j}=m}^{\sum_{i} \geq 1} \mathbf{x}_{k_{1}}(s+\mu) \cdots \mathbf{x}_{k_{j}}(s+\mu)\right. \\
&= e^{A(t+\mu)} \int^{t+\mu}\left[e^{-A s} \sum_{j=1}^{m} \frac{\partial}{\partial \mathbf{y}} \mathbf{F}^{(j)}(s+\mu, \mathbf{y}) \mathbf{R}^{(m+1-j)}(\mathbf{y})\right]_{N} \mathrm{~d} s \\
&\left.\quad-e^{-A s} \sum_{j=1}^{m} \frac{\partial}{\partial \mathbf{y}} \mathbf{F}^{(j)}(s, \mathbf{y}) \mathbf{R}^{(m+1-j)}(\mathbf{y})\right] \mathrm{d} s \\
&= e^{A(t+\mu)} \mathbf{F}^{(m+1)}(t+\mu, \mathbf{y}) . \\
& k_{1}+\cdots+k_{j}=m \\
& \mathbf{x}_{k_{1}}(s) \cdots \mathbf{x}_{k_{j}}(s)
\end{aligned}
$$

The proof is complete.

Theorem 4.1. For any $m \in \mathbb{N}$, Eq. (1.4) locally has a $C^{m}$ normal form as

$$
\frac{\mathrm{d} \boldsymbol{z}}{\mathrm{d} t}=A \boldsymbol{z}+\sum_{j=1}^{m} \varepsilon^{j} \boldsymbol{R}^{(j)}(\boldsymbol{z})+\mathcal{O}\left(\varepsilon^{m+1},\|\boldsymbol{z}\|^{m}\right)
$$

with the normal form transformation

$$
\boldsymbol{x}=\boldsymbol{h}(\boldsymbol{z})=\boldsymbol{z}+\sum_{i=1}^{m} \varepsilon^{i} \boldsymbol{F}^{(i)}(0, \boldsymbol{z}) .
$$

Proof. For given $m \in \mathbb{N}$, let

$$
\mathbf{H}(t, \mathbf{y})=e^{A t} \mathbf{y}+\sum_{i=1}^{m} \varepsilon^{i} \mathbf{F}^{(i)}(t, \mathbf{y}),
$$


then, by Lemma 4.1, we know that

$$
\mathbf{H}\left(t, e^{A \mu} \mathbf{y}\right)=e^{A(t+\mu)} \mathbf{y}+\sum_{i=1}^{m} \varepsilon^{i} \mathbf{F}^{(i)}(t+\mu, \mathbf{y}) .
$$

So the transformation (4.4) can be viewed as

$$
\mathbf{x}=\mathbf{h}(\mathbf{z})=\mathbf{H}(0, \mathbf{z})=\mathbf{H}\left(t, e^{-A t} \mathbf{z}\right) .
$$

Denoting by $\overline{\mathbf{z}}=e^{-A t} \mathbf{z}$, and taking (4.5) into Eq. (1.4), we obtain

$$
\begin{aligned}
& A \mathbf{x}+\varepsilon \mathbf{f}(\mathbf{x})=A \mathbf{x}+e^{A t} \frac{\mathrm{d} \overline{\mathbf{z}}}{\mathrm{d} t}+e^{A t} \sum_{i=1}^{m} \varepsilon^{i} \frac{\partial}{\partial t} \mathbf{F}^{(i)}(t, \overline{\mathbf{z}})+e^{A t} \sum_{i=1}^{m} \varepsilon^{i} \frac{\partial}{\partial \mathbf{y}} \mathbf{F}^{(i)}(t, \overline{\mathbf{z}}) \frac{\mathrm{d} \overline{\mathbf{z}}}{\mathrm{d} t} \\
& =A \mathbf{x}+e^{A t} \frac{\mathrm{d} \overline{\mathbf{z}}}{\mathrm{d} t}+\sum_{l=0}^{m-1}\left(\left.\frac{\mathrm{d}^{l}}{\mathrm{~d} \varepsilon^{l}} \mathbf{f}\left(e^{A t} \overline{\mathbf{z}}+e^{A t} \sum_{i=1}^{m} \varepsilon^{i} \mathbf{F}^{(i)}(t, \overline{\mathbf{z}})\right)\right|_{\varepsilon=0}\right) \varepsilon^{l+1} \\
& -\sum_{i=2}^{m} \varepsilon^{i} e^{A t} \sum_{j=1}^{i-1} \frac{\partial}{\partial \mathbf{y}} \mathbf{F}^{(j)}(t, \overline{\mathbf{z}}) \mathbf{R}^{(i-j)}(\overline{\mathbf{z}})-e^{A t} \sum_{j=1}^{m} \varepsilon^{j} \mathbf{R}^{(j)}(\overline{\mathbf{z}}) \\
& +e^{A t} \sum_{i=1}^{m} \varepsilon^{i} \frac{\partial}{\partial \mathbf{y}} \mathbf{F}^{(i)}(t, \overline{\mathbf{z}}) \frac{\mathrm{d} \overline{\mathbf{z}}}{\mathrm{d} t} \\
& =A \mathbf{x}+\varepsilon \mathbf{f}(\mathbf{x})+e^{A t}\left(i d+\sum_{i=1}^{m} \varepsilon^{i} \frac{\partial}{\partial \mathbf{y}} \mathbf{F}^{(i)}(t, \overline{\mathbf{z}})\right)\left(\frac{\mathrm{d} \overline{\mathbf{z}}}{\mathrm{d} t}-\sum_{j=1}^{m} \varepsilon^{j} \mathbf{R}^{(j)}(\overline{\mathbf{z}})\right) \\
& -\varepsilon \mathbf{f}\left(e^{A t} \overline{\mathbf{z}}+e^{A t} \sum_{i=1}^{m} \varepsilon^{i} \mathbf{F}^{(i)}(t, \overline{\mathbf{z}})\right) \\
& +\sum_{l=0}^{m-1}\left(\left.\frac{\mathrm{d}^{l}}{\mathrm{~d} \varepsilon^{l}} \mathbf{f}\left(e^{A t} \overline{\mathbf{z}}+e^{A t} \sum_{i=1}^{m} \varepsilon^{i} \mathbf{F}^{(i)}(t, \overline{\mathbf{z}})\right)\right|_{\varepsilon=0}\right) \varepsilon^{l+1} \\
& -\sum_{i=m+1}^{2 m} \varepsilon^{i} e^{A t} \sum_{j=1}^{i-1} \frac{\partial}{\partial \mathbf{y}} \mathbf{F}^{(j)}(t, \overline{\mathbf{z}}) \mathbf{R}^{(i-j)}(\overline{\mathbf{z}}) \\
& =A \mathbf{x}+\varepsilon \mathbf{f}(\mathbf{x})+\left(i d+\sum_{i=1}^{m} \varepsilon^{i} \frac{\partial}{\partial \mathbf{y}} \mathbf{F}^{(i)}(0, \mathbf{z})\right)\left(\frac{\mathrm{d} \mathbf{z}}{\mathrm{d} t}-A \mathbf{z}-\sum_{j=1}^{m} \varepsilon^{j} \mathbf{R}^{(j)}(\mathbf{z})\right) \\
& -\varepsilon \mathbf{f}\left(\mathbf{z}+\sum_{i=1}^{m} \varepsilon^{i} \mathbf{F}^{(i)}(0, \mathbf{z})\right)+\sum_{l=0}^{m-1}\left(\left.\frac{\mathrm{d}^{l}}{\mathrm{~d} \varepsilon^{l}} \mathbf{f}\left(\mathbf{z}+\sum_{i=1}^{m} \varepsilon^{i} \mathbf{F}^{(i)}(0, \mathbf{z})\right)\right|_{\varepsilon=0}\right) \varepsilon^{l+1} \\
& -\sum_{i=m+1}^{2 m} \varepsilon^{i} \sum_{j=1}^{i-1} \frac{\partial}{\partial \mathbf{y}} \mathbf{F}^{(j)}(0, \mathbf{z}) \mathbf{R}^{(i-j)}(\mathbf{z})
\end{aligned}
$$


which leads to

$$
\left(i d+\sum_{i=1}^{m} \varepsilon^{i} \frac{\partial}{\partial \mathbf{y}} \mathbf{F}^{(i)}(0, \mathbf{z})\right)\left(\frac{\mathrm{d} \mathbf{z}}{\mathrm{d} t}-A \mathbf{z}-\sum_{j=1}^{m} \varepsilon^{j} \mathbf{R}^{(j)}(\mathbf{z})\right)+\varepsilon^{m+1} \mathbf{S}_{m}(0, \mathbf{z}, \varepsilon)=0,
$$

that is to say

$$
\begin{aligned}
\frac{\mathrm{d} \mathbf{z}}{\mathrm{d} t} & =A \mathbf{z}+\sum_{j=1}^{m} \varepsilon^{j} \mathbf{R}^{(j)}(\mathbf{z})-\left(i d+\sum_{i=1}^{m} \varepsilon^{i} \frac{\partial}{\partial \mathbf{y}} \mathbf{F}^{(i)}(0, \mathbf{z})\right)^{-1} \varepsilon^{m+1} \mathbf{S}_{m}(0, \mathbf{z}, \varepsilon) \\
& =A \mathbf{z}+\sum_{j=1}^{m} \varepsilon^{j} \mathbf{R}^{(j)}(\mathbf{z})+\mathcal{O}\left(\varepsilon^{m+1},\|\mathbf{z}\|^{m}\right) .
\end{aligned}
$$

Then, the proof can be completed by combining the locally convergence from Theorem 3.1.

Remark 4.1. Based on the above discussions, it can be shown that the RG scheme we developed can be used to formulate the normal form for general differential equations by setting $\varepsilon=1$. Here, we should point out that the obtained RG normal form is, in general, different from the classical Poincaré-Dulac normal form up to any finite order. On the one hand, RG normal form is explicit, while the later one is implicit. On the other hand, it is not hard to see the fact that the RG normal form contains more terms rather than the latter one up to any order. For example, let us consider the following planar system:

$$
\left\{\begin{array}{l}
\frac{\mathrm{d} x_{1}}{\mathrm{~d} t}=x_{1}+x_{1}^{2} x_{2}+x_{1}^{3} x_{2}^{2}, \\
\frac{\mathrm{d} x_{2}}{\mathrm{~d} t}=-x_{2}+\delta x_{1} x_{2}+x_{2}^{2}+x_{1} x_{2}^{2},
\end{array}\right.
$$

where $\delta$ is a parameter. By direct calculation, one can get the RG normal form for (4.6) to order $\left(\left\|\left(y_{1}, y_{2}\right)\right\|^{2}\right)$

$$
\left\{\begin{array}{l}
\frac{\mathrm{d} y_{1}}{\mathrm{~d} t}=y_{1}+y_{1}^{2} y_{2}+y_{1}^{3} y_{2}^{2} \\
\frac{\mathrm{d} y_{2}}{\mathrm{~d} t}=-y_{2}+y_{1} y_{2}^{2}
\end{array}\right.
$$

while the corresponding Poincaré-Dulac normal form is

$$
\left\{\begin{array}{l}
\frac{d y_{1}}{d t}=y_{1} \\
\frac{d y_{2}}{d t}=-y_{2}
\end{array}\right.
$$


Further, the RG normal form for (4.6) to order $\left(\left\|\left(y_{1}, y_{2}\right)\right\|^{3}\right)$ can be obtained as

$$
\left\{\begin{array}{l}
\frac{\mathrm{d} y_{1}}{\mathrm{~d} t}=y_{1}+y_{1}^{2} y_{2}+y_{1}^{3} y_{2}^{2} \\
\frac{\mathrm{d} y_{2}}{\mathrm{~d} t}=-y_{2}+(1+\delta) y_{1} y_{2}^{2}
\end{array}\right.
$$

while the corresponding Poincaré-Dulac normal form is

$$
\left\{\begin{array}{l}
\frac{d y_{1}}{d t}=y_{1}+y_{1}^{2} y_{2} \\
\frac{d y_{2}}{d t}=-y_{2}+(1+\delta) y_{1} y_{2}^{2}
\end{array}\right.
$$

One can note that, in each order, the RG normal form contains more terms than the Poincaré-Dulac normal form. Especially, when $\delta=0$, even the RG normal form to order $\left(\left\|\left(y_{1}, y_{2}\right)\right\|^{2}\right)$ contains more terms than the Poincaré-Dulac normal form to order $\left(\left\|\left(y_{1}, y_{2}\right)\right\|^{3}\right)$.

\section{Approximating center manifold}

In this section, we try to investigate how does the renormalized solution asymptotically approach to the center manifold. We remark that Chiba considered this topic in [4] by means of KBM idea. Here, we carry out it in our strategy, and try to give more detailed results.

For simplicity, we consider the case when matrix $A$ in (1.4) can be represented as

$$
A=\left(\begin{array}{ll}
B & O \\
O & C
\end{array}\right),
$$

where $B$ is a $d \times d$ matrix having eigenvalues with zero real parts, $C$ is a $q \times q$ matrix having eigenvalues with negative real parts, $O$ denotes zero matrix, $d+q=n$. In what follows, for $\mathbf{x} \in \mathbb{C}^{n}$, we represent it as $\mathbf{x}=(\overline{\mathbf{x}}, \hat{\mathbf{x}})$ with $\overline{\mathbf{x}} \in \mathbb{C}^{d}, \hat{\mathbf{x}} \in \mathbb{C}^{q}$. Then, (1.4) can be rewritten as

$$
\left\{\begin{array}{l}
\dot{\overline{\mathbf{x}}}=B \overline{\mathbf{x}}+\varepsilon \overline{\mathbf{f}}(\overline{\mathbf{x}}, \hat{\mathbf{x}}), \\
\dot{\hat{\mathbf{x}}}=C \hat{\mathbf{x}}+\varepsilon \hat{\mathbf{f}}(\overline{\mathbf{x}}, \hat{\mathbf{x}}),
\end{array}\right.
$$

and it has local center manifold around the original point. Furthermore, we have following observations. 
Lemma 5.1. The $m$-th $R G$ equation can be rewritten as

$$
\left\{\begin{array}{l}
\frac{\mathrm{d} \bar{y}}{\mathrm{~d} t}=\sum_{j=1}^{m} \varepsilon^{j} \overline{\boldsymbol{R}}^{(j)}(\overline{\boldsymbol{y}}), \\
\frac{\mathrm{d} \hat{\boldsymbol{y}}}{\mathrm{d} t}=\sum_{j=1}^{m} \varepsilon^{j} \hat{\boldsymbol{R}}^{(j)}(\boldsymbol{y}),
\end{array}\right.
$$

and the m-th order approximate solution

$$
\boldsymbol{x}_{\varepsilon}=e^{A t} \boldsymbol{y}+\sum_{i=1}^{m} \varepsilon^{i} \boldsymbol{F}_{c}^{(i)}(t, \overline{\boldsymbol{y}})+\sum_{i=1}^{m} \varepsilon^{i} \boldsymbol{F}_{S}^{(i)}(t, \boldsymbol{y}),
$$

where $\overline{\boldsymbol{R}}^{(j)}(\overline{\mathbf{y}})$ is obtained by the first d parts of $\boldsymbol{R}^{(j)}(\boldsymbol{y}), \hat{\boldsymbol{R}}^{(j)}(\boldsymbol{y})$ is obtained by the last $q$ parts of $\boldsymbol{R}^{(j)}(\boldsymbol{y}), \boldsymbol{F}_{c}^{(j)}(t, \overline{\boldsymbol{y}})$ contains the oscillation terms in $e^{A t} \boldsymbol{F}^{(j)}(t, \boldsymbol{y}), \boldsymbol{F}_{S}^{(j)}(t, \boldsymbol{y})$ contains the exponential decay terms, and $\left.\hat{\boldsymbol{R}}^{(j)}(\boldsymbol{y})\right|_{\hat{y}=0}=0,\left.\boldsymbol{F}_{s}^{(j)}(t, \boldsymbol{y})\right|_{\hat{y}=0}=0, j=1, \ldots, m$.

Proof. Firstly, one can note that

$$
\begin{aligned}
\mathbf{R}^{(1)}(\mathbf{y})=\left[e^{-A t} \mathbf{f}\left(e^{A t} \mathbf{y}\right)\right]_{R}=\sum_{i=1}^{n}\left(\sum_{\alpha \in N_{r}^{i}} \mathbf{f}_{\alpha}^{i} \mathbf{y}^{\alpha}\right) \mathbf{e}_{i}=\left(\begin{array}{c}
\overline{\mathbf{R}}^{(1)}(\overline{\mathbf{y}}) \\
\hat{\mathbf{R}}^{(1)}(\mathbf{y})
\end{array}\right) \\
e^{A t} \mathbf{F}^{(1)}(t, \mathbf{y})=e^{A t} \int^{t}\left[e^{-A s} \mathbf{f}\left(e^{A s} \mathbf{y}\right)\right]_{N} \mathrm{~d} s=\sum_{i=1}^{n}\left(\sum_{\alpha \notin N_{r}^{i}} \mathbf{f}_{\alpha}^{i} \frac{e^{\langle\alpha, \Lambda\rangle t}}{\langle\alpha, \Lambda\rangle-\lambda_{i}} \mathbf{y}^{\alpha}\right) \mathbf{e}_{i} \\
=\mathbf{F}_{c}^{(1)}(t, \overline{\mathbf{y}})+\mathbf{F}_{s}^{(1)}(t, \mathbf{y})
\end{aligned}
$$

where $\mathbf{F}_{c}^{(1)}(t, \overline{\mathbf{y}})$ contains the oscillation terms in $e^{A t} \mathbf{F}^{(1)}(t, \mathbf{y}), \mathbf{F}_{\mathcal{S}}^{(1)}(t, \mathbf{y})$ contains the exponential decay terms which are also polynomial in $\hat{\mathbf{y}}$, and the lowest order in $\hat{\mathbf{y}}$ is at least 1 . By inductions, we assume that for $j \in\{1, \ldots, k\}$,

$$
\begin{aligned}
& \mathbf{R}^{(j)}(\mathbf{y})=\left(\begin{array}{c}
\overline{\mathbf{R}}^{(j)}(\overline{\mathbf{y}}) \\
\hat{\mathbf{R}}^{(j)}(\mathbf{y})
\end{array}\right), \\
& e^{A t} \mathbf{F}^{(j)}(t, \mathbf{y})=\mathbf{F}_{c}^{(j)}(t, \overline{\mathbf{y}})+\mathbf{F}_{s}^{(j)}(t, \mathbf{y}),
\end{aligned}
$$

where $\mathbf{F}_{c}^{(j)}(t, \overline{\mathbf{y}})$ contains the oscillation terms in $e^{A t} \mathbf{F}^{(j)}(t, \mathbf{y}), \mathbf{F}_{s}^{(j)}(t, \mathbf{y})$ contains the exponential decay terms, and $\left.\hat{\mathbf{R}}^{(j)}(\mathbf{y})\right|_{\hat{\mathbf{y}}=0}=0,\left.\mathbf{F}_{s}^{(j)}(t, \mathbf{y})\right|_{\hat{\mathbf{y}}=0}=0, j=1, \ldots, k$. Then

$$
\mathbf{R}^{(k+1)}(\mathbf{y})=\left[e^{-A t} \sum_{j=1}^{k} \frac{1}{j !} \partial^{j} \mathbf{f}\left(\mathbf{x}_{0}\right) \sum_{k_{1}+\cdots+k_{j}=k, k_{i} \geq 1} \mathbf{x}_{k_{1}} \cdots \mathbf{x}_{k_{j}}-\sum_{j=1}^{k} \frac{\partial}{\partial \mathbf{y}} \mathbf{F}^{(j)}(t, \mathbf{y}) \mathbf{R}^{(k+1-j)}(\mathbf{y})\right]_{R}
$$




$$
\begin{aligned}
& =\left[\left(\begin{array}{c}
e^{-B t} \\
e^{-C t}
\end{array}\right) \sum_{j=1}^{k} \frac{1}{j !} \partial^{j} \mathbf{f}\left(\mathbf{x}_{0}\right) \sum_{\substack{k_{1}+\cdots+k_{j}=k, k_{i} \geq 1 \\
\tau_{l}=c, s, l=1, \ldots, j}} \mathbf{F}_{\tau_{1}}^{\left(k_{1}\right)} \cdots \mathbf{F}_{\tau_{j}}^{\left(k_{j}\right)}-\sum_{j=1}^{k} \frac{\partial}{\partial \mathbf{y}} \mathbf{F}^{(j)}(t, \mathbf{y}) \mathbf{R}^{(k+1-j)}(\mathbf{y})\right]_{R} \\
& =\left(\begin{array}{l}
\overline{\mathbf{R}}^{(k+1)}(\overline{\mathbf{y}}) \\
\hat{\mathbf{R}}^{(k+1)}(\mathbf{y})
\end{array}\right)
\end{aligned}
$$

with $\left.\hat{\mathbf{R}}^{(k+1)}(\mathbf{y})\right|_{\hat{\mathbf{y}}=0}=0$, and

$$
\begin{aligned}
& e^{A t} \mathbf{F}^{(k+1)}(t, \mathbf{y}) \\
= & e^{A t} \int^{t}\left[e^{-A s} \sum_{j=1}^{k} \frac{1}{j !} \partial^{j} \mathbf{f}\left(\mathbf{x}_{0}\right) \sum_{\substack{k_{1}+\cdots+k_{j}=k, k_{i} \geq 1 \\
k_{1}}} \mathbf{x}_{k^{\prime}} \cdots \mathbf{x}_{k_{j}}-\sum_{j=1}^{k} \frac{\partial}{\partial \mathbf{y}} \mathbf{F}^{(j)}(s, \mathbf{y}) \mathbf{R}^{(k+1-j)}(\mathbf{y})\right]_{N} \mathrm{~d} s \\
= & e^{A t} \int^{t}\left[e^{-A s} \sum_{j=1}^{k} \frac{1}{j !} \partial^{j} \mathbf{f}\left(\mathbf{x}_{0}\right) \sum_{\substack{k_{1}+\cdots+k_{j}=k, k_{i} \geq 1 \\
\tau_{l}=c, s, l=1, \ldots, j}} \mathbf{F}_{\tau_{1}}^{k_{1}} \cdots \mathbf{F}_{\tau_{j}}^{k_{j}}-\sum_{j=1}^{k} \frac{\partial}{\partial \mathbf{y}} \mathbf{F}^{(j)}(s, \mathbf{y}) \mathbf{R}^{(k+1-j)}(\mathbf{y})\right]_{N} \mathrm{~d} s \\
= & \mathbf{F}_{c}^{(k+1)}(t, \overline{\mathbf{y}})+\mathbf{F}_{s}^{(k+1)}(t, \mathbf{y})
\end{aligned}
$$

with $\mathbf{F}_{c}^{(k+1)}(t, \overline{\mathbf{y}})$ the oscillation terms in $e^{A t} \mathbf{F}^{(j)}(t, \mathbf{y})$, and $\mathbf{F}_{S}^{(j)}(t, \mathbf{y})$ the exponential decay terms satisfying $\left.\mathbf{F}_{\mathcal{S}}^{(k+1)}(t, \mathbf{y})\right|_{\hat{\mathbf{y}}=0}=0$. Then the proof can be concluded.

Lemma 5.2. $\mathcal{S}=\left\{\boldsymbol{y} \in \mathbb{C}^{n} \mid \hat{\boldsymbol{y}}=0\right\}$ is an invariant manifold of system (5.2). Furthermore, $\mathcal{M} \triangleq \bigcup_{t \in \mathbb{R}} \boldsymbol{H}(t, \mathcal{S})=\boldsymbol{H}(0, \mathcal{S})$.

Proof. The first statement is obvious. For any given $\mu \in \mathbb{R}$ and $\mathbf{y} \in \mathcal{S}, e^{A \mu} \mathbf{y} \in \mathcal{S}$, therefore, by Lemma 4.1,

$$
\mathbf{H}(\mu, \mathbf{y})=\mathbf{H}\left(0, e^{A \mu} \mathbf{y}\right) \in \mathbf{H}(0, \mathcal{S}) \subset \bigcup_{t \in \mathbb{R}} \mathbf{H}(t, \mathcal{S})
$$

Hence the proof can be concluded by the arbitrary choice of $\mu \in \mathbb{R}$ and $\mathbf{y} \in \mathcal{S}$.

Theorem 5.1. There exist $\varepsilon_{0}>0$ and open set $\mathcal{U} \subset \mathbb{C}^{d}$ with $0 \in \mathcal{U}$, such that for any $\varepsilon \in\left(0, \varepsilon_{0}\right), \mathcal{M}_{0} \triangleq \boldsymbol{H}(0, \mathcal{U} \times\{0\})$ is the local center manifold of system

$$
\dot{\boldsymbol{z}}=A \boldsymbol{z}+\varepsilon \boldsymbol{f}(\boldsymbol{z})+\varepsilon^{m+1} \boldsymbol{S}_{m}(t, \boldsymbol{y}, \varepsilon) .
$$

Furthermore, $\mathcal{M}_{0}$ is an $\mathcal{O}\left(\varepsilon^{m+1}\right)$ approximation of a local center manifold $\mathcal{M}_{\varepsilon}$ of (5.1). 
Proof. Firstly, for any $\mathbf{z}_{0} \in \mathcal{M}$, there exists $\mathbf{y}_{0} \in \mathcal{S}$, such that $\mathbf{z}_{0}=\mathbf{H}\left(0, \mathbf{y}_{0}\right)$, therefore, the solution $\mathbf{z}(t)$ of (5.3) with $\mathbf{z}(0)=\mathbf{z}_{0}$ can be, in fact, presented as

$$
\mathbf{z}(t)=\mathbf{H}(t, \mathbf{y}(t)) \in \mathbf{H}(0, \mathcal{S})=\mathcal{M},
$$

this implies that $\mathcal{M}$ is an invariant manifold of (5.3).

Secondly, consider the equations

$$
\mathbf{z}=\left(\begin{array}{c}
\overline{\mathbf{z}} \\
\hat{\mathbf{z}}
\end{array}\right)=\left(\begin{array}{c}
\overline{\mathbf{y}} \\
\mathbf{0}
\end{array}\right)+\sum_{i=1}^{m} \varepsilon^{i} \mathbf{F}_{c}^{(i)}(0, \overline{\mathbf{y}}),
$$

or

$$
\left\{\begin{array}{l}
\overline{\mathbf{z}}=\overline{\mathbf{y}}+\sum_{i=1}^{m} \varepsilon^{i} \overline{\mathbf{F}}_{c}^{(i)}(0, \overline{\mathbf{y}}) \triangleq \overline{\mathbf{h}}(\overline{\mathbf{y}}, \varepsilon), \\
\hat{\mathbf{z}}=\sum_{i=1}^{m} \varepsilon^{i} \hat{\mathbf{F}}_{c}^{(i)}(0, \overline{\mathbf{y}}) .
\end{array}\right.
$$

Then, by the implicit function theorem, there exist some $\varepsilon_{0}>0$ and open set $0 \in$ $\mathcal{U} \subset \mathbb{C}^{d}$, such that the first equation in (5.4) can be written as $\overline{\mathbf{y}}=\overline{\mathbf{h}}^{-1}(\overline{\mathbf{z}}, \varepsilon)=\overline{\mathbf{z}}+$ $\mathcal{O}\left(\|(\overline{\mathbf{z}}, \varepsilon)\|^{2}\right)$, which is analytic in $(\overline{\mathbf{z}}, \varepsilon)$ in $\mathcal{U} \times\left(0, \varepsilon_{0}\right)$. Hence

$$
\hat{\mathbf{z}}=\sum_{i=1}^{m} \varepsilon^{i} \hat{\mathbf{F}}_{c}^{(i)}\left(0, \overline{\mathbf{h}}^{-1}(\overline{\mathbf{z}}, \varepsilon)\right)=\mathcal{O}\left(\|\overline{\mathbf{z}}\|^{2}, \varepsilon\right), \quad \overline{\mathbf{z}} \in \overline{\mathbf{h}}\left(\mathcal{U},\left(0, \varepsilon_{0}\right)\right), \quad \varepsilon \in\left(0, \varepsilon_{0}\right),
$$

this means

$$
\mathcal{M}_{0}=\mathbf{H}(0, \mathcal{U} \times\{0\})=\left\{\mathbf{z} \in \mathbb{C}^{n} \mid \hat{\mathbf{z}}=\sum_{i=1}^{m} \varepsilon^{i} \hat{\mathbf{F}}_{c}^{(i)}\left(0, \overline{\mathbf{h}}^{-1}(\overline{\mathbf{z}}, \varepsilon)\right), \overline{\mathbf{z}} \in \overline{\mathbf{h}}\left(\mathcal{U},\left(0, \varepsilon_{0}\right)\right)\right\}
$$

is the local center manifold of (5.3).

Furthermore, it is easy to note that, there exists an open set $\mathcal{V} \subset \overline{\mathcal{V}} \subset \mathcal{U}$ with $0 \in \mathcal{V}$, such that $\mathbf{H}(0, \mathcal{V} \times\{0\})$ is normally hyperbolic and (5.3) is an $\mathcal{O}\left(\varepsilon^{m+1}\right)$ perturbation of (5.1), then, by Fenichel theorem [8], we can get a normally hyperbolic manifold $\mathcal{M}_{\varepsilon}$ of (5.1), which is an $\mathcal{O}\left(\varepsilon^{m+1}\right)$ perturbation of $\mathbf{H}(0, \mathcal{V} \times\{0\})$, with no loss of generality, we can express $\mathcal{M}_{\varepsilon}$ as

$$
\hat{\mathbf{x}}=\sum_{i=1}^{m} \varepsilon^{i} \hat{\mathbf{F}}_{c}^{(i)}\left(0, \overline{\mathbf{h}}^{-1}(\overline{\mathbf{x}})\right)+\varepsilon^{m+1} \mathbf{h}_{0}(\overline{\mathbf{x}}, \varepsilon), \quad \overline{\mathbf{x}} \in \overline{\mathbf{h}}\left(\mathcal{V},\left(0, \varepsilon_{0}\right)\right), \quad \varepsilon \in\left(0, \varepsilon_{0}\right) .
$$

Taking (5.6) into (5.1), and combining with the property of (5.5), one can easily conclude that $\mathbf{h}_{0}(\overline{\mathbf{x}}, \varepsilon)=\mathcal{O}\left(\|\overline{\mathbf{x}}\|^{2}\right)$, which implies that $\mathcal{M}_{\varepsilon}$ is indeed the local center manifold of (5.1). 
Corollary 5.1. There exist some $\varepsilon_{0}>0$ and an open set $\mathcal{V} \subset \mathbb{C}^{d}$ with $0 \in \mathcal{V}$, such that, for any $\varepsilon \in\left(0, \varepsilon_{0}\right)$,

$$
\operatorname{dist}\left(z(t), \mathcal{M}_{\varepsilon}\right)<M\left(\varepsilon^{m+1}+e^{-\alpha t}\right), \quad t \in[0, T / \varepsilon],
$$

where $\boldsymbol{z}(t)$ is the solution of (5.3) with $\boldsymbol{z}(0)=\boldsymbol{z}_{0} \in \mathbf{H}\left(0, \mathcal{V} \times \mathbb{C}^{q}\right),-\alpha$ is the biggest real part of eigenvalues of $C$, and $T, M>0$ are constants only depending on $\varepsilon_{0}$ and $\mathcal{V}$.

Proof. From Theorem 5.1, we only need to prove that for $\varepsilon \in\left(0, \varepsilon_{0}\right]$

$$
\operatorname{dist}\left(\mathbf{z}(t), \mathcal{M}_{0}\right)<M e^{-\alpha t}, \quad t \in[0, T / \varepsilon]
$$

In fact, we can consider the solution $\mathbf{w}(t)$ of (5.3) on $\mathcal{M}_{0}$ such that $\mathbf{w}(0)=H\left(0, \mathbf{y}_{1}\right)$ with $\overline{\mathbf{y}}_{1}=\overline{\mathbf{y}}_{0}, \hat{\mathbf{y}}_{1}=0$, here $\mathbf{y}_{0} \in \mathcal{V}$ satisfies $\mathbf{H}\left(0, \mathbf{y}_{0}\right)=\mathbf{z}_{0}$. Then

$$
\begin{aligned}
& \mathbf{z}(t)=e^{A t} \mathbf{y}+\sum_{i=1}^{m} \varepsilon^{i} \mathbf{F}_{c}^{(i)}(t, \overline{\mathbf{y}})+\sum_{i=1}^{m} \varepsilon^{i} \mathbf{F}_{s}^{(i)}(t, \mathbf{y}), \\
& \mathbf{w}(t)=e^{A t}\left(\begin{array}{c}
\overline{\mathbf{y}} \\
0
\end{array}\right)+\sum_{i=1}^{m} \varepsilon^{i} \mathbf{F}_{c}^{(i)}(t, \overline{\mathbf{y}}) \in \mathcal{M}_{0}
\end{aligned}
$$

where $\mathbf{y}(t)$ is the solution of RG equation (5.2) with $\mathbf{y}(0)=\mathbf{y}_{0}$. Based on the discussions in above sections, for any $\varepsilon \in\left(0, \varepsilon_{0}\right]$, there exists $T>0$ such that

$$
\mathbf{w}(t) \in \mathcal{M}_{0}, \quad t \in[0, T / \varepsilon],
$$

and furthermore, there exists $M>0$ depending on $\varepsilon_{0}$ and $\mathcal{V}$ such that

$$
\|\mathbf{z}(t)-\mathbf{w}(t)\| \leq\left\|e^{A t}\left(\begin{array}{c}
0 \\
\hat{\mathbf{y}}
\end{array}\right)\right\|+\sum_{i=1}^{m} \varepsilon^{i}\left\|\mathbf{F}_{s}^{(i)}(t, \mathbf{y})\right\| \leq M e^{-\alpha t}, \quad t \in[0, T / \varepsilon] .
$$

So (5.7) holds and the proof is complete.

\section{Acknowledgments}

This work is supported by NSFC grant (Nos. 11771177, 12171197), China Automobile Industry Innovation and Development Joint Fund (No. U1664257), Program for Changbaishan Scholars of Jilin Province and Science and Technology Development Project of Jilin Province (No. YDZJ202101ZYTS141, 20190201132JC). 


\section{References}

[1] L. Y. Chen, N. Goldenfeld and Y. Oono, Renormalization group theory for global asymptotic analysis, Phys. Rev. Lett. 73(10) (1994), 1311-1315.

[2] L. Y. Chen, N. Goldenfeld and Y. Oono, Renormalization group and singular perturbations: multiple scales, boundary layers, and reductive perturbation theory, Phys. Rev. E 543(1) (1996), 376-394.

[3] H. Chiba, $C^{1}$ approximation of vector fields based on the renormalization group method, SIAM J. Appl. Dyn. Syst. 7(3) (2008), 895-932.

[4] H. Chiba, Approximation of center manifolds on the renormalization group method, J. Math. Phys. 49(10) (2008), 102703.

[5] H. Chiba, Extension and unification of singular perturbation methods for ODEs based on the renormalization group method, SIAM J. Appl. Dyn. Syst. 8(3) (2009), 1066-1115.

[6] R. E. Lee DeVilla, A. Harkin, M. Holzer, K. Josić and T. J. Kaper, Analysis of a renormalization group method and normal form theory for perturbed ordinary differential equations, Physica D 237(8) (2008), 1029-1052.

[7] S. I. Ei, K. Fujii and T. Kunihiro, Renormalization-group method for reduction of evolution equations: invariant manifolds and envelopes, Ann. Physics 280(2) (2000), 236-298.

[8] N. Fenichel, Geometric singular perturbation theory for ordinary differential equations, J. Differential Equations 31(1) (1979), 53-98.

[9] N. Goldenfeld, B. P. Athreya and J. A. Dantzig, Renormalization group approach to multiscale modelling in materials science, J. Stat. Phys. 125 (2006), 1019-1027.

[10] S. J. Gustafson and I. M. Sigal, Mathematical concepts of quantum mechanics, SpringerVerlag, 2009.

[11] E. Kirkinis, The Renormalization Group: a perturbation method for the graduate curriculum, SIAM Rev. 54(2) (2012), 374-388.

[12] W. L. Li and S. Y. Shi, Singular perturbed renormalization group theory and its application to highly oscillatory problems, Discrete Contin. Dyn. Syst. Series B 23(4) (2018), 1819 1833.

[13] I. Moise and R. Temam, Renormalization group method. Applications to Navier-Stokes equation, Discrete Contin. Dyn. Syst. 6(1) (2000), 191-200.

[14] I. Moise and M. Ziane, Renormalization group method. Applications to partial differential equations, J. Dynam. Differential Equations 13(2) (2001), 275-321.

[15] R. E. O'Malley and E. Kirkinis, Examples illustrating the use of renormalization techniques on singularly perturbed differential equations, Stud. Appl. Math. 122(2) (2009), 105-122.

[16] R. E. O'Malley and E. Kirkinis, A combined renormalization group-multiple scale method for singularly perturbed problems, Stud. Appl. Math. 124(4) (2010), 383-410.

[17] M. Petcu, R. Temam and D. Wirosoetisno, Renormalization group method applied to the primitive equations, J. Differential Equations 208(1) (2005), 215-257. 
[18] J. A. Sanders, F. Verhulst and J. Murdock, Averaging methods in nonlinear dynamical systmes, Texts in Applied Mathematics 59, Springer-Verlag, 2007.

[19] A. Sarkar, J. K. Bhattacharjee, S. Chakraborty and D. B. Banerjee, Center or limit cycle: renormalization group as a probe, Eur. Phys. J. D 64 (2011), 479-489.

[20] R. M. Temam and D. Wirosoetisno, On the solutions of the renormalized equations at all orders, Adv. Differential Equations 8(8) (2003), 1005-1024.

[21] K. Walid and S. Ablou, On the renormalization group approach to perturbation theory for PDEs, Ann. Henri Poincaré, 11(6) (2010), 1007-1021.

[22] M. Ziane, On a certain renormalization group method, J. Math. Phys. 41(5) (2003), 32903299.

[23] R. Zhou, S. Y. Shi, and W. L. Li, Renormalization group approach to boundary layer problems, Commun. Nonlinear Sci. Numer. Simul. 71 (2019), 220-230. 